\title{
Title: The complete chloroplast genome of Onobrychis gaubae (Fabaceae-Papilionoideae): comparative analysis with related IR-lacking clade species
}

\author{
Mahtab Moghaddam \\ Tarbiat Modares University \\ Atsushi Ohta \\ Kyoto University \\ Motoki Shimizu \\ Kyoto University \\ Ryohei Terauchi \\ Kyoto University \\ Shahrokh Kazempour-Osaloo ( $\square$ skosaloo@modares.ac.ir) \\ Tarbiat Modares University
}

\section{Research Article}

Keywords: Onobrychis gaubae, genomes, population genetics

Posted Date: March 15th, 2021

DOI: https://doi.org/10.21203/rs.3.rs-290026/v1

License: (c) (i) This work is licensed under a Creative Commons Attribution 4.0 International License.

Read Full License 


\section{Abstract}

Plastid genome sequences provide valuable markers for surveying the evolutionary relationships and population genetics of plant species. In the present study, the complete plastid genome of Onobrychis gaubae, endemic to Iran, was sequenced using Illumina paired-end sequencing and was compared with previously known genomes of the IRLC species of legumes. The 0 . gaubae plastid genome was 123,645 bp in length and included a large single-copy (LSC) region of $81,034 \mathrm{bp}$, a small single-copy (SSC) region of $13,788 \mathrm{bp}$ and one copy of the inverted repeat $\left(\mathrm{IR}_{\mathrm{b}}\right)$ of $28,823 \mathrm{bp}$. The genome encoded 110 genes, including 76 protein-coding genes, 30 transfer RNA (tRNA) genes and four ribosome RNA (rRNA) genes and possessed 89 simple sequence repeats (SSRs) and 28 repeated structures with the highest proportion in the LSC. Comparative analysis of the chloroplast genomes across IRLC revealed three hotspot genes $(y c f 1, y c f 2, c / p \mathrm{P})$ which could be used as molecular markers for resolving phylogenetic relationships and species identification. IRLC plastid genomes also showed multiple gene losses and inversions. Phylogenetic analyses revealed that $O$. gaubae is closely related to Hedysarum. The complete O. gaubae genome is a valuable resource for investigating evolution of Onobrychis species and can be used to identify related species.

\section{Introduction}

Chloroplast is a vital organelle in plant cells that has an important role in plant carbon fixation and numerous metabolic pathways ${ }^{1,2}$. In angiosperms, the chloroplast genome (plastome) typically has a circular structure that ranges from 120 to $180 \mathrm{~kb}$ in length. Plastomes mostly exhibit a quadripartite structure in which a pair of inverted repeats (IRa and IRb; usually around $25 \mathrm{~kb}$, but can vary from 7 to 88 $\mathrm{kb}$ each) separate the large single-copy (LSC, ca. $80 \mathrm{~kb}$ ) and the small single-copy (SSC, ca. $20 \mathrm{~kb}$ ) regions ${ }^{1,2}$. Most plastomes encodes 80 protein-coding genes primarily involved in photosynthesis and other biochemical processes along with 30 tRNA and 4 rRNA genes ${ }^{3,4}$. In contrast to mitochondrial and nuclear genomes, the plastomes across seed plants are highly conserved with respect to gene content, structure and organization ${ }^{5,6}$. However, mutations including duplication, rearrangements, and losses have been reported at the genome and gene levels among some angiosperm lineages, including Asteraceae ${ }^{7}$, Campanulaceae $^{8}$, Onagraceae ${ }^{9}$, Fabaceae $^{10}$ and Geraniaceae ${ }^{11}$.

Fabaceae (legumes) is the third largest family of angiosperms which shows the most extensive structural variation ${ }^{12}$. Currently accepted classification of the legumes based on plastid gene matK includes six subfamilies: Caesalpinioideae, Cercidoideae, Detarioideae, Dialioideae, Duparquetioideae, and Papilionoideae ${ }^{13}$. Gene content and gene order in plastomes of subfamilies are highly conserved and similar to the ancestral angiosperm genome organization except for Papilionoideae, which exhibits numerous rearrangements and gene/intron losses and have a smaller genome ${ }^{5}$. In this subfamily, a loss of one of the $\mathrm{IR}^{14}$, the presence of many repetitive sequences ${ }^{15}$ and the presence of a localized hypermutable region ${ }^{15,16}$ have been documented. The Papilionoideae is further divided into six major clades: the Genistoids, Dalbergioids, Mirbelioids, Millettioids, Robinioids and the inverted-repeat lacking 
clade (IRLC) ${ }^{14}$. IRLC is the largest legume lineage which contains over 4000 species in 52 genera and eight tribes ${ }^{14,17-19}$. Recently, with the advent of next generation sequencing (NGS) technology, plastomes of several taxa from different tribes in this clade have been sequenced. The majority of IRLC plastomes sequenced to date were restricted to the tribes Fabeae, Trifolieae and Caraganeae. Thus it is essential to investigate the members from other lineages to better understand plastome evolution within the IRLC, and more broadly within Papilionoideae. In the tribe Hedysareae ${ }^{20}$ with nine genrea, the plastomes of some Hedysarum species and only one species of Onobrychis have been reported. In the present study, the complete plastome of $O$. gaubae Bornm. belonging to Hedysareae was sequenced (GenBank accession number: ???). Onobrychis has more than 130 species and is the second largest genus after Hedysarum and mainly found throughout temperate and subtropical regions of Eurasia, $\mathrm{N}$ and NE Africa ${ }^{21}$. Onobrychis gaubae is a polymorphic species restricted to the southern slopes of Alborz mountain range, $\operatorname{Iran}^{22,23}$.

The main goal of this study is to assemble the chloroplast genome of O. gaubae, and to annotate the genome and characterize its structure to provide new genomic resource of this species. We also performed comparative analyses of the genome and phylogenetic reconstruction to evaluate the sequence divergence in the plastomes across the IR-lacking clade.

\section{Results And Discussion}

\section{General features of the 0 . gaubae plastid genome}

It was previously reported that the plastomes of Papilionoideae, particularly IR-loss clade, are not conserved in their genomic structure in terms of gene order and gene content and exhibit numerous rearrangements and gene/intron losses $5,24,25$. The plastid genome of $O$. gaubae with 123,645 bp in length and having only one copy of the IR region, which is in accordance with the reports and its genome structure is similar to those of other IRLC species. In this context, the lack of $r p s 16$ and $r p / 22$ genes and intron 1 of $c / p P$ in the plastome of 0 . gaubae should be noted; these genes, are absent from the chloroplast genomes of entire IRLC ${ }^{24,26}$. The assembled chloroplast genome of 0 . gaubae contained 110 genes, including 76 protein-coding genes, 30 transfer RNA (tRNA) genes and four ribosome RNA (rRNA) genes (Fig. 1, Table 1). The LSC ( 81,066 bp), SSC (13,777 bp) and IR (28,802 bp) regions along with the locations of 110 genes in the chloroplast genome are shown in Fig. 1. A total of 16 genes contained a single intron, whereas $y c f 3$ exhibits two introns (Supplementary Table S1). The rps12 gene is a transsplicing gene which does not have introns in the 3 '-end. The trnK-UUU has the largest intron encompassing the matK gene, with $2,495 \mathrm{bp}$, whereas the intron of trnL-UAA is the smallest (542 bp). The overall GC content of the $O$. gaubae chloroplast genome sequence was $35.1 \%$, which is consistent with other IRLC species, whose plastomes have GC-contents ranging from 33.6-35.1\% (Table 2). Different GC content occurs in the LSC (34.4\%), SSC (30.8\%) and IR (39.2\%) regions (Supplementary Table S2). Higher $\mathrm{GC}$ content was usually detected in the IRs compared to the other regions of plastome, which is mainly due to the presence of rRNA genes (rrn23, rrn16, rrn5, rrn4.5) with high GC content (50\%-56.4\%) in IRs 6,27 . 
The GC content of the protein-coding regions of 0 . gaubae chloroplast genome comprised $36.02 \%$. Within these regions, the GC contents for the first, second and third positions of the codons were $42.4 \%, 36.1 \%$ and $29.5 \%$, respectively. 
Table 1

Genes predicted in the chloroplast genome of O. gaubae. The number of asterisks after the gene names indicates the number of introns contained in the genes.

\begin{tabular}{|c|c|c|}
\hline $\begin{array}{l}\text { Category of } \\
\text { genes }\end{array}$ & Group of genes & Name of genes \\
\hline \multirow[t]{5}{*}{ Self-replication } & $\begin{array}{l}\text { Large subunit of } \\
\text { ribosomal proteins }\end{array}$ & $r p / 14, r p / 16 *, r p / 2 *, r p / 20, r p / 23, r p / 32, r p / 33, r p / 36$ \\
\hline & $\begin{array}{l}\text { Small subunit of } \\
\text { ribosomal proteins }\end{array}$ & $\begin{array}{l}r p s 2, r p s 3, r p s 4, r p s 7, r p s 8, r p s 11, r p s 12 *, r p s 14, r p s 15 \\
r p s 18, r p s 19\end{array}$ \\
\hline & $\begin{array}{l}\text { DNA-dependent RNA } \\
\text { polymerase }\end{array}$ & $r p o \mathrm{~A}, r p o \mathrm{~B}, r p o \mathrm{C} 1 *, r p o \mathrm{C} 2$ \\
\hline & Ribosomal RNA genes & $r r n 16 \mathrm{~S}, r r n 23 S, r r n 4.5 \mathrm{~S}, r r n 5 \mathrm{~S}$ \\
\hline & Transfer RNA genes & 30 trn genes (5 contain an intron) \\
\hline \multirow[t]{6}{*}{$\begin{array}{l}\text { Genes for } \\
\text { photosynthesis }\end{array}$} & $\begin{array}{l}\text { Subunits of NADH- } \\
\text { dehydrogenase }\end{array}$ & $\begin{array}{l}n d h \mathrm{~A}^{*}, n d h \mathrm{~B}^{*}, n d h \mathrm{C}, n d h \mathrm{D}, n d h \mathrm{E}, n d h \mathrm{~F}, n d h \mathrm{G}, n d h \mathrm{H}, n d h \mathrm{l}, \\
n d h \mathrm{~J}, n d h \mathrm{~K}\end{array}$ \\
\hline & $\begin{array}{l}\text { Subunits of } \\
\text { photosystem I }\end{array}$ & psaA, psaB, psaC, psal, psaJ \\
\hline & $\begin{array}{l}\text { Subunits of } \\
\text { photosystem II }\end{array}$ & $\begin{array}{l}p s b \mathrm{~A}, p s b \mathrm{~B}, p s b \mathrm{C}, p s b \mathrm{D}, p s b \mathrm{E}, p s b \mathrm{~F}, p s b \mathrm{H}, p s b \mathrm{l}, p s b \mathrm{~J} \\
p s b \mathrm{~K}, p s b \mathrm{~L}, p s b \mathrm{M}, p s b \mathrm{~N}, p s b \mathrm{~T}, p s b \mathrm{Z}\end{array}$ \\
\hline & $\begin{array}{l}\text { Subunits of } \\
\text { cytochrome } b / f \\
\text { complex }\end{array}$ & pet $\mathrm{A}$, pet $\mathrm{B}^{*}$, pet $\mathrm{D} *$, pet $\mathrm{G}$, pet $\mathrm{L}$, pet $\mathrm{N}$ \\
\hline & $\begin{array}{l}\text { Subunits of ATP } \\
\text { synthase }\end{array}$ & $\operatorname{atp\mathrm {A}}, \operatorname{atp\mathrm {B}}, \operatorname{atp\mathrm {E}}, \operatorname{atp\mathrm {F}^{*}}, \operatorname{atp\mathrm {H}}$, atpl \\
\hline & Subunit of rubisco & $r b c \mathrm{~L}$ \\
\hline \multirow[t]{5}{*}{ Other genes } & Maturase K & matK \\
\hline & $\begin{array}{l}\text { Envelope membrane } \\
\text { protein }\end{array}$ & cemA \\
\hline & $\begin{array}{l}\text { Subunit of Acetyl-CoA- } \\
\text { carboxylase }\end{array}$ & $\mathrm{accD}$ \\
\hline & $\begin{array}{l}\text { C-type cytochrome } \\
\text { synthesis gene }\end{array}$ & $\operatorname{ccs} \mathrm{A}$ \\
\hline & Protease & $c / p P^{*}$ \\
\hline $\begin{array}{l}\text { Genes of } \\
\text { unkown } \\
\text { function }\end{array}$ & $\begin{array}{l}\text { Conserved } \\
\text { hypothetical } \\
\text { chloroplast } \\
\text { open reading frames }\end{array}$ & $y c f 1, y c f 2, y c f 4, y c f 3 \star \star$ \\
\hline
\end{tabular}


Table 2

Chloroplast genome information from sampled IRLC species and the newly assembled O. gaubae. LSC: Large Single Copy, SSC: Small Single Copy, IR: Inverted Repeat.

\begin{tabular}{|llllll|}
\hline Species & Size (bp) & LSC (bp) & SSC (bp) & IR (bp) & GC (\%) \\
\hline Astragalus mongholicus & 123,582 & 80,986 & 13,773 & 28,823 & $34.1 \%$ \\
\hline Caragana microphylla & 130,029 & 85,436 & 14,106 & 30,487 & $34.3 \%$ \\
\hline Carmichaelia australis & 122,805 & 80,588 & 14,074 & 28,143 & $34.3 \%$ \\
\hline Cicer arietinum & 125,319 & 82,583 & 13,820 & 28,916 & $33.9 \%$ \\
\hline Glycyrrhiza glabra & 127,943 & 84,714 & 14,187 & 29,042 & $34.2 \%$ \\
\hline Hedysarum semenovii & 123,407 & 80,288 & 13,679 & 29,440 & $34.9 \%$ \\
\hline Lens culinaris & 122,967 & 81,659 & 13,833 & 27,604 & $34.4 \%$ \\
\hline Lessertia frutescens & 122,700 & 80,698 & 13,750 & 28,252 & $34.2 \%$ \\
\hline Medicago sativa & 125,330 & 83,756 & 13,383 & 28,191 & $34 \%$ \\
\hline Melilotus albus & 127,205 & 84,279 & 13,806 & 29,120 & $33.6 \%$ \\
\hline Meristotropis xanthioides & 127,735 & 84,629 & 14,150 & 28,956 & $34.2 \%$ \\
\hline Onobrychis gaubae & 123,645 & 81,066 & 13,777 & 28,802 & $35.1 \%$ \\
\hline Oxytropis bicolor & 122,461 & 80,170 & 14,017 & 28,274 & $34.2 \%$ \\
\hline Tibetia liangshanensis & 123,372 & 79,916 & 13,513 & 29,943 & $34.7 \%$ \\
\hline Wisteria floribunda & 130,561 & 87,193 & 14,127 & 29,628 & $34.4 \%$ \\
\hline
\end{tabular}

\section{Codon usage bias}

The total coding DNA sequences (CDSs) were 81,121 bp in length and encoded 75 genes including 24,765 codons which belonged to 61 codon types. Codon usage was calculated for the protein-coding genes present in the 0 . gaubae cp genome. Phenylalanine was the most abundant amino acid, whereas arginine showed the least abundance in this species (Supplementary Table S3). Most protein-coding genes employ the standard ATG as the initiator codon. Among the $O$. gaubae protein-coding genes, three genes used alternative start codons; ACG for $p s b \mathrm{~L}, \mathrm{GTG}$ for $r p s 8$ and ACG for $n d h \mathrm{D}$.

The chloroplast genomes of the IRLC were analyzed for their codon usage frequency according to sequences of protein-coding genes and relative synonymous codon usage (RSCU). RSCU is an important indicator to measure codon usage bias in coding regions. This value is the ratio between the actual observed values of the codon and the theoretical expectations. If RSCU $=1$, codon usage is unbiased; if RSCU > 1, specific codon frequency is higher than other synonymous codons, otherwise, the frequency is 
low $^{28,29}$. The total number of codons among protein-coding genes in the IRLC species varies from 20,381 codons in Hedysarum taipeicum (as the smallest number) to 24,765 codons in 0 . gaubae. The most often used synonymous codon was AUU, encoding isoleucine, and the least used was CGC/CGG, encoding arginine (Supplementary Table S4). In the IRLC, the standard AUG codon was usually the start codon for the majority of protein-coding genes and UAA was the most frequent stop codon among three stop codons. Methionine (AUG) and tryptophan (UGG) showed RSCU $=1$, indicating no codon bias for these two amino acids. The highest RSCU value was for UUA ( 2.04) in leucine and the lowest was GGC ( 0.35 ) in glycine. Leucine preferred six codon types (UUA, UUG, CUU, CUC, CUA, and CUG) and actually showed $A$ or $T(U)$ bias in all synonymous codons (Supplementary Table S4). The result of distributions of codon usage in the IRLC species showed that RSCU $>1$ was recorded for most codons that ended with an A or a U, except for UUG codon, resulting in the bias for A/T bases. As well as, more codons with the RSCU value less than one, ended with base $C$ or G. So, there is high A/U preference in the third codon of the IR-loss clade coding regions, which is a common phenomenon in cp genomes of higher plants ${ }^{30}$.

\section{Analysis of repeats}

Repeat analysis of the 0 . gaubae plastome identified 28 repeat structures with lengths ranging from 30 bp to $81 \mathrm{bp}$. These structures included 12 palindromic repeats with lengths in the range of 33-50 bp, 11 forward repeats of 30-81 bp, four reverse repeats which varied from $30 \mathrm{bp}$ to $31 \mathrm{bp}$ and one complementary repeats with a length of $30 \mathrm{bp}$ (Supplementary Table S5). Among the 28 repeats, $75 \%$ are located in the LSC region, $14.28 \%$ in the SSC region and $10.71 \%$ in the IR region. Also, most of the repeats $(60.71 \%$ ) were found in the intergenic spacer regions (IGS), $25 \%$ were distributed in coding region (psaA, psal, $p s b J$, trnR-UCU, trnS-UGA) and $14.28 \%$ were located in the introns (ndhA and $y c f 3$ ). In the majority of the studied IRLC species, the most frequently observed repeats were forward, then palindromic, and the least was the reverse (Fig. 2). The forward type was the most abundant repeat with length in the range 30-50 bp in all the IRLC species. The longest repeats were also of the forward type, with length of $560 \mathrm{bp}$ were detected in the Hedysarum taipeicum, followed by Vicia sativa of $517 \mathrm{bp}$ and Caragana microphylla of $455 \mathrm{bp}$, which were much longer than other species studied. Furthermore, in the IRLC, repeat sequences involved in genome rearrangement, were mainly distributed in non-coding regions (IGS). Repeat structures induce indels and substitutions resulting in the mutation hotspot in the reconfiguration of genome ${ }^{6}$; therefore, these repeats can provide valuable information for phylogenetic and population studies ${ }^{29}$.

Simple sequence repeats (SSRs), or microsatellites, are a type of tandem repeat sequences which contain 1-6 nucleotide repeat units and have wide distribution throughout the genome ${ }^{29,31}$. Accordingly, microsatellites play a crucial role in the genome recombination and rearrangement. These nucleotide motifs show a high level of polymorphism that can be widely used for phylogenetic analysis, population genetics and species authentication ${ }^{29,32}$. A total of 89 SSRs were detected in the 0 . gaubae plastome, which were composed by a length of at least $10 \mathrm{bp}$. Among them, 53 (59.55\%) were mono-repeats, 21 (23.55\%) were di-repeats, $10(11.23 \%)$ were tri-repeats and five $(5.61 \%)$ were tetra-repeats. No penta- and hexanucleotide SSRs existed in 0 . gaubae genome (Supplementary Table S6). These SSR loci were located primarily in the LSC region (68.53\%), followed by the SSC region (16.85\%) and IR (14.6\%) 
(Fig. 3A). In the mononucleotide repeats, A/T motifs were the most abundant but no G/C motif was detected in the cp genome. Likewise, the majority of the dinucleotides and trinucleotides were found to be particularly rich in AT sequences. Therefore, the AT richness in the SSRs of the chloroplast genome of $O$. gaubae is consistent with the results of previous studies ${ }^{27,29}$ which have shown that in the cp genome, SSRs generally composed of polythymine (poly $\mathrm{T}$ ) or polyadenine (poly A) repeats ${ }^{33}$. The number of SSRs in the cp genomes (cpSSRs) ranged from 68 (Vicia sativa and Lens culinaris) to 151 (Melilotus albus) across the IRLC species (Fig. 3B). The mononucleotide repeats (P1) were identified at a much higher frequency, which varied from 45 (Tibetia liangshanensis, Glycyrrhiza glabra) to 93 (Melilotus albus). In all cases, the P1s were AT-rich (Fig. 3C). Strong AT bias in SSR loci was also observed in other legumes such as Vigna radiate ${ }^{34}$, Arachis hypogaea ${ }^{35}$ and Stryphnodendron adstringens ${ }^{27}$ which, like other plastomes of species, may contribute to the bias in base composition ${ }^{6}$. The results showed that SSR loci of LSC regions appeared more frequently than in SSC or IR regions, which may be hypothesized that this phenomenon is relevant to the lack of one IR region in IR-loss clade. In general, cpSSRs show abundant variation and might provide useful information for detecting intra- and interspecific polymorphisms at the population level ${ }^{31,33}$.

\section{Divergent hotspots in the IRLC chloroplast genomes}

The average nucleotide diversity $(\mathrm{Pi})$ among the protein-coding genes of 19 species of the IRLC was estimated to be 0.05736 . Furthermore, comparison of nucleotide diversity in the LSC, SSC and IR regions indicated that the IR region exhibits the highest nucleotide diversity (0.11549) and the SSC region shows the least (0.04132). We detected three hyper-variable regions with Pi values $>0.1$ among the IRLC species; $y c f 1$ and $y c f 2$ from IR region and $c / p P$ from LSC region (Fig. 4). These genes might be undergoing rapid nucleotide substitution in IR-loss clade at the genus and species levels. Among these, ycf1 encoding a protein of 1800 amino acids has the highest nucleotide diversity $(0.18745)$. ycf1 gene is more variable than matK gene and it can be useful for molecular systematics at low taxonomic levels ${ }^{36,37}$. Numerous studies $^{14,18,38}$ analyzed the phylogenetic reconstructions of IRLC species at various taxonomic levels based on different fragments of plastid coding genes such as matK, $n d h \mathrm{~F}$ and $r b c \mathrm{~L}$, the nuclear ribosomal ITS and the combined sequences of these genes/spacers. We could use the highly variable regions acquired from this study to develop the potential phylogenetic markers which can be useful for species authentication and reconstruction of phylogeny within different tribes/genera of IR-loss clade in further studies.

The non-synonymous $(\mathrm{Ka})$ to synonymous $(\mathrm{Ks})$ rate ratio $(\mathrm{Ka} / \mathrm{Ks})$ was estimated for 75 protein-coding genes across the 28 IRLC species analyzed (Supplementary Table S7). In general, the Ka/Ks values were lower than 0.5 for almost all genes. The $y c f 4$ gene which is involved in regulating the assembly of the photosystem I complex had the highest nonsynonymous rate, 0.165691 , while the $y c f 1$ gene with unknown functions had the highest synonymous rate, 0.181067 . The $\mathrm{Ka} / \mathrm{Ks}$ ratio (denoted as $\omega$ ) is widely used as an estimator of selective pressure for protein-coding genes. An $\omega>1$ indicates that the gene is affected by positive selection, $\omega<1$ indicates purifying (negative) selection, and $\omega$ close to 1 indicates neutral mutation ${ }^{39}$. In present study, the Ka/Ks ratio was calculated to be 0 for $p s b \mathrm{~L}$ gene which 
encodes one of the subunits of photosystem II. The Ka/Ks ratio indicates purifying selection in 73 protein-coding genes. The highest $\mathrm{Ka} / \mathrm{Ks}$ ratio which indicates positive selection was observed in $a c c \mathrm{D}$ gene which encodes a subunit of the acetyl-CoA carboxylase (ACCase) enzyme. Some studies have investigated whether selective pressure is acting on a particular protein-coding gene in different genera/tribes of IR-loss clade. For instance, tests for positive selection suggested that Lathyrus, Pisum and Vavilovia, all belonging tribe Fabeae, have undergone adaptive evolution in the $y c f 4$ gene $^{15,16}$.

Legumes chloroplast genome, and in particular IRLC, have regions with high mutation rates, including rps16-accD-psal-ycf4-cemA region. rps16 gene was lost from cpDNA in the common ancestor of the IRloss clade ${ }^{15}$. accD coding region was completely absent in the Trifolium subgenus Trifolium and has nuclear copies in Medicago truncatula and Cicer arietinum ${ }^{25}$. Three consecutive genes psal-ycf4-cemA is situated in a local mutation hotspot and has been lost in some species of Lathyrus ${ }^{15,16}$.

\section{Comparative analysis of genome structure}

We compared whole chloroplast genome sequences of different taxa of IRLC to analyze gene order and content. We found that, similar to other plant species, the gene coding regions were more conserved than the noncoding regions (Fig. 5). High nucleotide variations were observed across IRLC for the proteincoding regions $y c f 1, y c f 2$ and $c / p P$. Similar results were also obtained from calculation of nucleotide diversity (Pi). Papilionoideae, in particular the IRLC, displays structural variations which provide informative characters to increase phylogenetic resolution and make the taxon an excellent model for genome evolution studies ${ }^{5,25}$. The plastomes of several members of the IRLC have regions with significant variation and rearrangement and accelerated mutation rates, including loss of introns from rps 12 and $c / p P$ genes ${ }^{24}$, absence of $r p s 16$ gene $^{26}$ and transfer/loss of rp/22 to the nucleus ${ }^{24}$. Numerous studies have also shown some other rearrangements in some IRLC taxa, such as loss of accD gene in six species of Trifolium ${ }^{10,25}$, loss of $r p / 23$ and $r p / 33$ genes in some species of Lathyrus, Pisum and Vicia ${ }^{32}$ and loss of $y c f 4$ gene in some species of Lathyrus and Pisum ${ }^{15,16}$. As revealed in other studies, there are several reasons for occurrence of rearrangements in the plastome, such as the lack of one IR region, variable IR region size and many tandemly repeated sequences ${ }^{40}$. For example, the loss of the $r p s 16$ gene was probably due to the presence of a nuclear rps16 copy, which contributed to pseudogenization of the plastid copy ${ }^{41}$. Likewise, the lack or expansion of the $a c c D$ gene was explained by the presence of tandemly repeated sequences ${ }^{6,15}$.

\section{Plastid RNA editing prediction}

RNA editing is one of the post-transcriptional events which converts cytidine $(\mathrm{C})$ to uridine $(\mathrm{U})$ or $\mathrm{U}$ to $\mathrm{C}$ at specific sites of RNA molecules and modifies the genetic information from the genome in the plastids and mitochondria of land plants. RNA editing serves as a mechanism to correct missense mutations of genes by inserting, deleting and modifying nucleotides in a transcript ${ }^{42}$. RNA editing sites of 0 . gaubae plastid genes were predicted using Prep-CP prediction tool (Supplementary Table S8). In total, 58 editing sites were present in 19 chloroplast protein-coding genes and all of the editing sites were C-to-U conversions (Supplementary Table S8). Among them, nine editing sites, the highest number, were found 
in the region encoding $n d h \mathrm{~B}$ gene followed by seven editing sites in petB (Fig. 6). There were six editing sites detected each in $n d h \mathrm{~A}$ and $r p o \mathrm{~B}$ genes. $a c c \mathrm{D}, n d h \mathrm{G}$ and petD had three editing sites, and $n d h \mathrm{D}$ and $n d h \mathrm{~F}$ had two editing sites. Two editing sites were also found in ccsA, matK and rpoC1 genes. The remaining seven genes had only one editing site. The results showed that $n d h$ genes exhibited the most abundant editing sites which were nearly $39.6 \%$ of the total editing sites. In flowering plants, the highest number of plastid editing sites was found in the $n d h$ group genes ${ }^{42}$. Moreover, the $n d h$ genes encoding for a thylakoid Ndh complex, have been lost or pseudogenized in different species of algae, bryophytes, pteridophytes, gymnosperms, monocots, eudicots, magnoliids, and protists ${ }^{43-45}$. The RNA editing is probably important for the NDH protein complex function and may also lead to improved photosynthesis and display positive selection during evolution ${ }^{42}$.

\section{Phylogenetic relationships}

Phylogenetic relationships within the IRLC were reconstructed using the representative taxa (27 species from different tribes) and two species as outgroup based on 75 protein-coding genes of their chloroplast genomes. The total concatenated alignment length from the 75 protein-coding genes was $87,455 \mathrm{bp}$. The reconstructed phylogeny is in agreement with previous studies $5,14,25,46$ indicated that IRLC was monophyletic and consisted of several clades. As shown in the previous studies, Glycyrrhiza+ Meristotropis were monophyletic, along with the tribe Wisterieae was sister to the rest of the IRLC ${ }^{19,47}$. Then, there are two major clades: clade I and II (Fig. 7). Clade I comprises tribes Caraganeae ${ }^{17}$, Hedysarea $\mathrm{e}^{20}$ and Colutea ${ }^{18}$ as well as genera Oxytropis and Astragalus. Our results showed a close relationship between $O$. gaubae and $O$. viciifolia and Hedysarum species and confirmed $O$. gaubae phylogenetic position in the tribe Hedysareae. Furthermore, our plastid DNA analyses which are consistent with the previous study ${ }^{46}$, show that Oxytropis is sister to the tribe Coluteae. In clade II, tribe Cicereae is the basal branch and formed a sister group relationship with the paraphyletic Trifolieae and the monophyletic tribe Fabeae. The results of the present study suggest that there is no conflict between the phylogeny made by whole cp genome and that inferred by individual gene datasets. Therefore, a phylogenetic reconstruction for IR-loss clade species studied here showed that plastid genome database will be a helpful resource for molecular phylogeny at the higher taxonomic level (generic to tribal rank).

\section{Materials And Methods}

\section{Chloroplast DNA extraction and sequencing}

The young leaves of $O$. gaubae were collected in the natural habitat in northwestern Tehran, Iran. Genomic DNA was extracted from dried leaves using a DNeasy Plant Kit (Qiagen) according to the manufacturer's instructions. DNA quality and quantity were confirmed using $1 \%$ agarose gel electrophoresis and the resulting DNA was sequenced using the Illumina HiSeq-2000 platform at Iwate Biotechnology Research Center. The paired-end libraries were constructed according to the manufacturer's protocol (Illumina Inc., San Diego, CA). In total, 43,189,861 paired-end reads each comprising 100-bp sequence were obtained. 


\section{Genome Assembly and Annotation}

Using the complete plastid genome of Astragalus nakaianus (KR296789) as the reference, the paired-end reads of $O$. gaubae were filtered and assembled in to a complete plastome using Fast-Plast (https://github.com/mrmckain/Fast-Plast) ${ }^{48}$. Gaps in the cpDNA sequences were filled by PCR amplification and Sanger sequencing. The de novo assembled chloroplast genomes were annotated by $\mathrm{GeSeq}^{49}$. We used the online tRNAscan-SE service ${ }^{50}$ to improve the identification of tRNA genes. To detect the number of matched reads and the depth of coverage, raw reads were remapped to the assembled plastomes with Bowtie $2^{51}$ as implemented in Geneious v.9.0.2. The entire chloroplast genome sequences of 0 . gaubae was deposited in GenBank (Accession Number:???).

\section{Codon usage}

Codon usage was determined for all protein-coding genes. The codon usage analysis was performed in the web server Bioinformatics (https://www.bioinformatics.org/sms2/codon_usage.html). Furthermore, the relative synonymous codon usage (RSCU) values were determined with MEGA $X^{52}$, which was used to reveal the characteristics of the variation in synonymous codon usage.

\section{Characterization of repeat sequences}

REPuter was used to identify forward repeats, reverse sequences, complementary and palindromic sequences, with a minimal size of $30 \mathrm{bp}$, hamming distance of 3 and over $90 \%$ identity. Simple sequence repeats (SSRs) were detected using the microsatellite identification tool MISA (available online: http://pgrc.ipk-gatersleben.de/misa/misa.html). The minimum numbers of the SSR motifs were 10, 5, 4, 3,3 and 3 for mono-, di-, tri-, tetra-, penta-, and hexanucleotide repeats, respectively.

\section{Divergent hotspots identification and synonymous (Ks) and non-synonymous (Ka) substitution rate analysis}

To assess the nucleotide diversity $(\mathrm{Pi})$ among the plastid genomes of the representative species of the IRLC, the whole chloroplast genome sequences were aligned using MAFFT ${ }^{53}$ on XSEDE v.7.402 in CIPRES Science Gateway ${ }^{54}$. A sliding window analysis was conducted to determine the nucleotide diversity of the chloroplast genome using DnaSP v.6.12 software ${ }^{55}$. The window length was set to $800 \mathrm{bp}$ and the step size was $200 \mathrm{bp}$. Furthermore, the protein-coding regions of the 28 chloroplast genomes were used to evaluate evolutionary rate variation within the IRLC. Thus, we aligned the 75 protein-coding genes separately using MAFFT and then estimated the synonymous (Ks) and non-synonymous (Ka) substitution rates, as well as their ratio $(\mathrm{Ka} / \mathrm{Ks})$ using DnaSP v.6.12 software.

\section{Genome comparison}

To investigate divergence in chloroplast genomes, identity across the whole $\mathrm{cp}$ genomes was visualized using the mVISTA viewer in the Shuffle-LAGAN mode ${ }^{56}$ among the 17 IRLC accessions using Glycyrrhiza glabra as the reference. 


\section{Prediction of potential RNA editing sites}

Thirty-five protein-coding genes of $O$. gaubae were used to predict potential RNA editing sites using the Predictive RNA Editor for Plants (PERP)-Cp web server (http://prep.unl.edu) ${ }^{57}$ with a cutoff value of 0.8 .

\section{Phylogenetic reconstruction}

Seventy-five protein-coding genes were recorded from 27 species within IRLC, as well as from two outgroups (Robinia pseudoacacia L. and Lotus japonicus (Regel) K.Larsen). All genes sequences were obtained from GenBank (Supplementary Table S9). The concatenated data were analyzed using maximum likelihood and Bayesian inference methodologies. Prior to maximum likelihood and Bayesian analyses, a general time reversible and gamma distribution $(G T R+G)$ model was selected using the MrModeltest2. $2^{58}$ under the Akaike Information Criteria (AIC) ${ }^{59}$. Maximum likelihood analyses were performed using the online phylogenetic software W-IQ-TREE ${ }^{60}$ available at http://iqtree.cibiv.univie.ac.at. Nodes supports were calculated via rapid bootstrap analyses with 5000 replicates. Bayesian inference was performed using MrBayes v.3.2 in the CIPRES ${ }^{54}$ with the following settings: Markov chain Monte Carlo simulations for 5,000,000 generations with four incrementally heated chains, starting from random trees and sampling one out of every 1,000 generations. The first $25 \%$ of the trees were regarded as burnins. The remaining trees were used to construct a $50 \%$ majority-rule consensus tree and to estimate posterior probabilities. Posterior probabilities (PP) $>0.95$ were considered as significant support for a clade.

\section{Conclusions}

In this study, the complete plastome sequence of 0 . gaubae $(123,645 \mathrm{bp})$ was determined. The gene contents and gene orientation of $O$. gaubae plastome are similar to those found in the plastid genome of other IRLC species. Comparison of plastomes across IRLC showed that the coding regions are more conserved than non-coding regions and IR is more conserved than LSC and SSC regions. The present study also analyzed genetic information in the IRLC plastomes including the distribution and location of repeat sequences and SSRs, codon usage, RNA editing prediction, hotspot regions and phylogenomic analysis. Moreover, we identified three hotspot genes $(y c f 1, y c f 2, c / p P)$ which provided sufficient genetic information for species identification and phylogenetic reconstruction of the IRLC species. Finally, the data obtained from this study could provide a useful resource for further research on tribe Hedysareae and also IR-loss clade at the genomic scale.

\section{Declarations}

Competing Interests:

The authors declare no competing interests.

\section{Author contributions}


M.M. and S.K.O. conceived the idea, designed the study and carried out the plant sampling; M.M., A.O. and M.S. extracted chloroplast DNA for next generation sequencing, A.O. and M.S. assembled the genome, M.M. and A.O. performed the manual genome annotation, M. M. performed the phylogenetic and computational analyses, M.M. wrote the paper. R.T. and S.K.O. edited and reviewed the paper.

\section{Data Availability}

The complete chloroplast sequence generated and analyzed during the current study are available in GenBank (accession number are described in the text).

\section{References}

1. Jansen, R. K., Ruhlman, T. A. Plastid Genomes of Seed Plants. In Genomics of Chloroplasts and Mitochondria 103-126, https://doi.org/10.1007/978-94-007-2920-9_5 (Springer, Dordrecht, 2012).

2. Ruhlman, T. A., and Jansen, R. K. The plastid genomes of flowering plants in Chloroplast Biotechnology: Methods and Protocols (ed. Maliga P.) 3-38 (Springer, Humana Press, 2014).

3. Jansen, R. K. et al. Methods for obtaining and analyzing whole chloroplast genome sequences. Methods Enzymol. 395,348-384 (2005).

4. Bock, R. Structure, function, and inheritance of plastid genomes in Cell and Molecular Biology of Plastids (ed. Bock, R.) 29-63, https://doi.org/10.1007/4735_2007_0223 (Springer-Verlag Berlin Heidelberg, 2007).

5. Schwarz, E.N. et al. Plastid genome sequences of legumes reveal parallel inversions and multiple losses of rps16 in papilionoids. J Syst Evol. 53, 458-468 (2015).

6. Asaf, S. et al. Comparative analysis of complete plastid genomes from wild soybean (Glycine soja) and nine other Glycine species. PLOS ONE 12(8): e0182281 (2017).

7. Kim, K.J., Choi, K.S., Jansen, R. K. Two chloroplast DNA inversions originated simultaneously during the early evolution of the sunflower family (Asteraceae). Mol Biol Evol. 22(9): 1783 \pm 92 (2005).

8. Haberle, R.C., Fourcade, H.M., Boore, J.L., Jansen, R.K. Extensive rearrangements in the chloroplast genome of Trachelium caeruleum are associated with repeats and tRNA genes. J. Mol. Evol. 66(4): $350 \pm 61$ (2008).

9. Greiner, S. et al. The complete nucleotide sequences of the five genetically distinct plastid genomes of Oenothera, subsection Oenothera: I. Sequence evaluation and plastome evolution. Nucleic Acids Res. 36(7): $2366 \pm 78$ (2008).

10. Cai, Z. et al. Extensive reorganization of the plastid genome of Trifolium subterraneum (Fabaceae) is associated with numerous repeated sequences and novel DNA insertions. J. Mol. Evol. 67(6): $696 \pm 704$ (2008).

11. Guisinger, M.M., Kuehl, J.V., Boore, J.L., Jansen, R.K. Extreme reconfiguration of plastid genomes in the angiosperm family Geraniaceae: rearrangements, repeats, and codon usage. Mol. Biol. Evol. 28(1): $583 \pm 600$ (2011). 
12. Palmer, J.D., Osorio, B., Thompson, W.F. Evolutionary significance of inversions in legume chloroplast DNAs. Curr. Genet. 14, 65-74 (1988).

13. Legume Phylogeny Working Group. Legume phylogeny and classification in the $21^{\text {st }}$ century: A new subfamily classification of the Leguminosae based on a taxonomically comprehensive phylogeny. Taxon. 66,44-77 (2017).

14. Wojciechowski, M.F., Lavin, M., Sanderson, M.J. A phylogeny of legumes (Leguminosae) based on analysis of the plastid matk gene resolves many well-supported subclades within the family. Am. J. Bot. 91, 1846-1862 (2004).

15. Magee, A.M. et al. Localized hypermutation and associated gene losses in legume chloroplast genomes. Genome Research. 20, 1700-1710 (2010).

16. Moghaddam M., Kazempour-Osaloo S. Extensive survey of the $y c f 4$ plastid gene throughout the IRLC legumes: Robust evidence of its locus and lineage specific accelerated rate of evolution, pseudogenization and gene loss in the tribe Fabeae. PLOS ONE 15(3), e0229846 (2020).

17. Duan, L. et al. A molecular phylogeny of Caraganeae (Leguminosae, Papilionoideae) reveals insights in to new generic and infrageneric delimitations. PhytoKeys. 70, 111-137 (2016).

18. Moghaddam, M., Kazempour Osaloo, S., Hosseiny, H., Azimi, F. Phylogeny and divergence times of the Coluteoid clade with special reference to Colutea (Fabaceae) inferred from nrDNA ITS and two cpDNAs, matK and rp/32-trnL(UAG) sequences data. Plant Biosyst. 6, 1082-1093 (2017).

19. Compton, J.A. et al. The Callerya Group redefined and Tribe Wisterieae (Fabaceae) emended based on morphology and data from nuclear and chloroplast DNA sequences. PhytoKeys. 125, 1-112 (2019).

20. Amirahmadi, A., Kazempour Osaloo, S., Moein, F., Kaveh, A., Maassoumi, A.A. Molecular systematic of the tribe Hedysareae (Fabaceae) based on nrDNA ITS and plastid trnL-F and matK sequences. Plant Syst. Evol. 300, 729-747 (2014).

21. Lock, J.M. Tribe Hedysarae in Legumes of the world. (eds. Lewis G, Schrire B, Mackinder B, Lock M) 489-495 (Royal Botanical Gardens, Kew 2005).

22. Rechinger KH. Tribus Hedysareae Papilionaceae II, Flora Iranica. (ed. Rechinger KH) 387-464 (Akademische Druckund Verlagsanstalt, Graz 1984).

23. Kaveh, A., Kazempour-Osaloo, S., Amirahmadi, A., Maassoumi, A., Schneeweiss, G. Systematics of Onobrychis sect. Heliobrychis (Fabaceae): morphology and molecular phylogeny revisited. Plant Syst. Evol.305, 33-48 (2019).

24. Jansen, R.K., Wojciechowski, M.F., Sanniyasi, E., Lee, S.B., Daniell, H. Complete plastid genome sequence of the chickpea (Cicer arietinum) and the phylogenetic distribution of $r p s 12$ and $c / p P$ intron losses among legumes (Leguminosae). Mol. Phylogenet. Evol. 48, 1204-1217 (2008).

25. Sabir, J. et al. Evolutionary and biotechnology implications of plastid genome variation in the inverted-repeat-lacking clade of legumes. Plant Biotechnol. J. 12, 743-754 (2014). 
26. Doyle, J. J., Doyle, J. L., Palmer, J. D. Multiple independent losses of two genes and one intron from legume chloroplast genomes. Syst. Bot. 20, 272-294 (1995).

27. Souza, U.J.B.d. et al.The complete chloroplast genome of Stryphnodendron adstringens(Leguminosae - Caesalpinioideae): comparative analysis with related Mimosoid species. Sci. Rep. 9, 14206 (2019).

28. Sharp, P.M., Li ,W.H. The codon adaptation index-a measure of directional synonymous codon usage bias, and its potential applications. Nucleic Acids Res. 15(3), 1281-1295 (1987).

29. Li, X. et al.Comparison of Four Complete Chloroplast Genomes of Medicinal and Ornamental MeconopsisSpecies: Genome Organization and Species Discrimination. Sci. Rep. 9, 10567 (2019).

30. Li, C.J., Wang, R.N., Li, D.Z. Comparative analysis of plastid genomes within the Campanulaceae and phylogenetic implications. PLoS ONE 15(5), e0233167 (2020).

31. Powell, W., Morgante, M., Mcdevitt, R.,Vendramin, G.G., Rafalski, J.A. Polymorphic Simple Sequence Repeat Regions in Chloroplast Genomes-Applications to the Population-Genetics of Pines. PNAS. 92(17), 7759 \pm 63 (1995).

32. Lei, W. et al. Intraspecific and heteroplasmic variations, gene losses and inversions in the chloroplast genome of Astragalus membranaceus. Sci. Rep. 22, 6:21669 (2016).

33. Zong, D. et al. Comparative analysis of the complete chloroplast genomes of seven Populus species: Insights into alternative female parents of Populus tomentosa. PLoS ONE 14(6), e0218455 (2019).

34. Tangphatsornruang, S. et al. The Chloroplast Genome Sequence of Mungbean (Vigna radiata) Determined by High-throughput Pyrosequencing: Structural Organization and Phylogenetic Relationships. DNA Res. 17(1), 11 \pm 22 (2010).

35. Yin, D. et al. Development of chloroplast genome resources for peanut (Arachis hypogaea L.) and other species of Arachis. Sci. Rep. 7, 11649 (2017).

36. Neubig, K. M. et al. Phylogenetic utility of $y c f 1$ in orchids: a plastid gene more variable than matK. Plant Syst. Evol. 277, 75-84 (2009).

37. Dong, W. et al. ycf1, the most promising plastid DNA barcode of land plants. Sci. Rep. 5, 8348 (2015).

38. Schaefer, $\mathrm{H}$. et al. Systematics, biogeography, and character evolution of the legume tribe Fabeae with special focus on the middle-atlantic island lineages. BMC Evol. Biol. 12, 250 (2012).

39. Yang, Z., Wong, W.S.W., Nielsen, R. Bayes empirical bayes inference of aminoacid sites under positive selection. Mol Biol Evol. 22, 1107-1118 (2005).

40. Wicke, S., Schneeweiss, G.M., dePamphilis, C.W., Muller, K.F., Quandt, D. The evolution of the plastid chromosome in land plants: Gene content, gene order, gene function. Plant Mol. Biol. 76, 273-297 (2011).

41. Keller, J. et al. The evolutionary fate of the chloroplast and nuclear rps16 genes as revealed through the sequencing and comparative analyses off our novel legume chloroplast genomes from Lupinus. DNA Res. 24(4), 343-358 (2017). 
42. He, P. et al.Abundant RNA editing sites of chloroplast protein-coding genes in Ginkgo bilobaand an evolutionary pattern analysis. BMC Plant Biol. 16, 257 (2016).

43. Blazier , J. , Guisinger, M. M. , Jansen, R. K. Recent loss of plastid-encoded $n d h$ genes within Erodium (Geraniaceae). Plant Mol. Biol. 76, 263 - 272 (2011).

44. Ruhlman , T. et al. NDH expression marks major transitions in plant evolution and reveals coordinate intracellular gene loss. BMC Plant Biol. 15, 100 (2015).

45. Sanderson, M.J. et al. Exceptional reduction of the plastid genome of saguaro cactus (Carnegiea gigantea): Loss of the ndh gene suite and inverted repeat. Am. J. Bot. 102(7), 1115-27 (2015).

46. Su, C. et al. Chloroplast phylogenomics and character evolution of eastern Asian Astragalus (Leguminosae): Tackling the phylogenetic structure of the largest genus of flowering plants in Asia. Mol Phylogenet Evol. 156, 107025. (2021).

47. Duan, L. et al. Chloroplast Phylogenomics Reveals the Intercontinental Biogeographic History of the Liquorice Genus (Leguminosae: Glycyrrhiza). Front. Plant Sci. 11, 793 (2020).

48. McKain, M.R., Wilson, M. mrmckain/Fast-Plast: Fast-Plast v.1.2.8. Version v.1.2.8. (2018). https://github.com/mrmckain/Fast-Plast.

49. Tillich, M. et al. GeSeq - versatile and accurate annotation of organelle genomes. Nucleic Acids Res.45, W6-W11 (2017).

50. Schattner, P., Brooks, A.N., Lowe, T.M. The tRNA scan-SE, snoscan and snoGPS web servers for the detection of tRNAs and snoRNAs. Nucleic Acids Res. 33, W686 WW (2005).

51. Langmead, B. Salzberg, S. L. Fast gapped-read alignment with Bowtie 2. Nat. Methods 9, 357-359 (2012).

52. Kumar, S. et al. MEGA X: molecular evolutionary genetics analysis across computing platforms. Mol. Biol. Evol. 35(6), 1547-1549 (2018).

53. Katoh, K., Standley, D. M. MAFFT Multiple Sequence Alignment Sofware Version 7: Improvements in Performance and Usability. Mol. Biol. Evol. 30, 772-780 (2013).

54. Miller, M.A., Pfeiffer, W., Schwartz, T. Creating the CIPRES science gateway for inference of large phylogenetic trees. Proceedings of the Gateway Computing Environments Workshop (GCE); New Orleans, Louisiana. (2010).

55. Rozas, J. et al. DnaSP 6: DNA Sequence Polymorphism Analysis of Large Data Sets. Mol. Biol. Evol. 34, 3299-3302 (2017).

56. Frazer, K. A., Pachter, L., Poliakov, A., Rubin, E. M., Dubchak, I. VISTA: computational tools for comparative genomics. Nucleic Acids Res. 32, W273-W279 (2004).

57. Mower, J.P. The PREP suite: Predictive rna editors for plant mitochondrial genes, chloroplast genes and user-defined alignments. Nucleic Acids Res. 37, W253-W259 (2009).

58. Nylander, J.A.A. MrModeltest v2. Program distributed by the author. Uppsala: Evolutionary Biology Centre, Uppsala University. (2004). 
59. Posada, D., Buckley, T.R. Model selection and model averaging in phylogenetics: advantages of Akaike information criterion and Bayesian approaches over likelihood ratio tests. Syst. Biol. 53,793808 (2004).

60. Trifinopoulos, J., Nguyen, L.T., Haeseler, A., Minh, B.Q. W-IQ-TREE: a fast online phylogenetic tool for maximum likelihood analysis. Nucleic Acids Res. 44(W1), W232-W235 (2016).

Figures

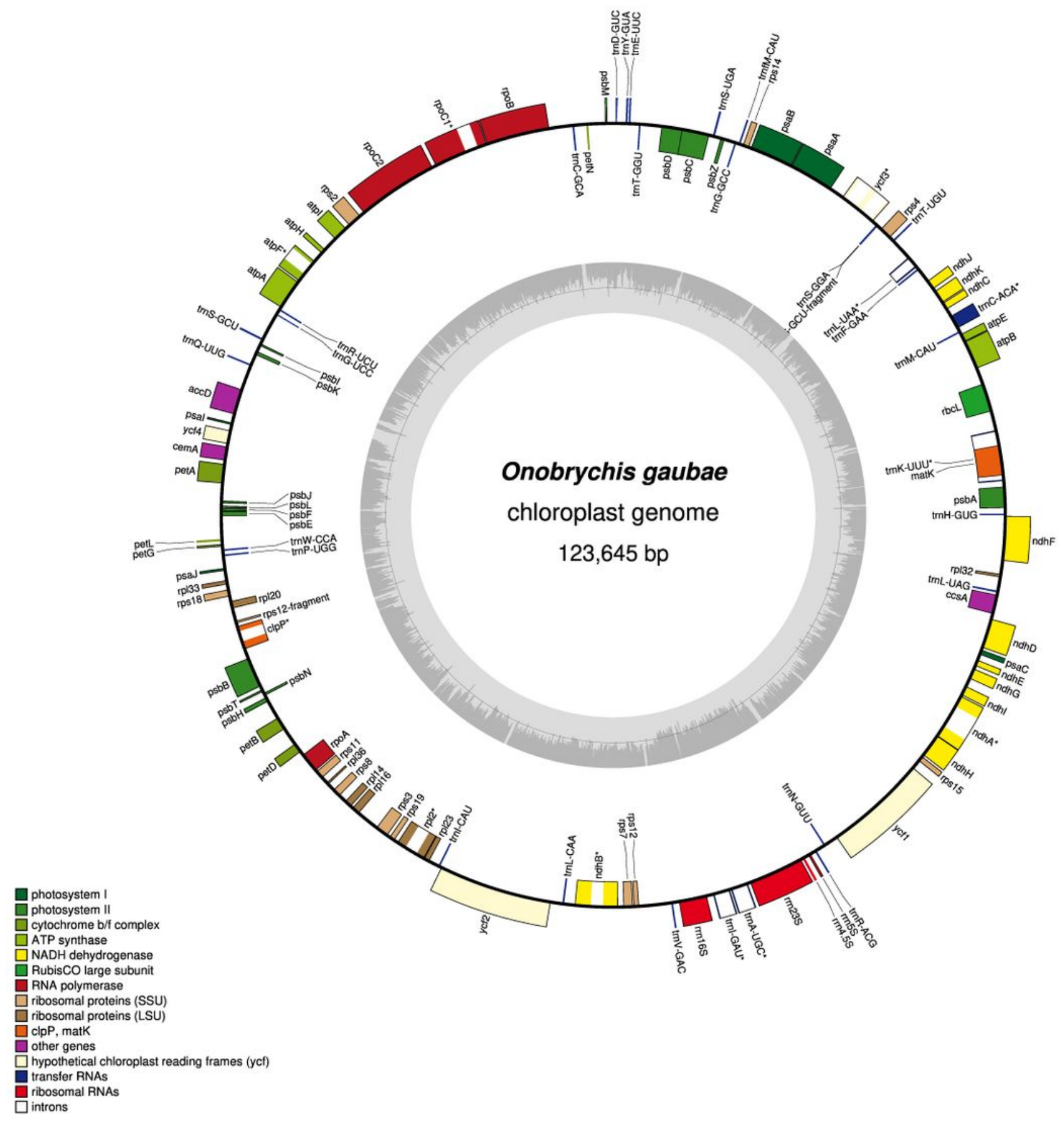


Figure 1

Gene map of the 0. gaubae chloroplast genome. The genes drawn outside and inside of the circle are transcribed in clockwise and counterclockwise directions, respectively. Genes were colored based on their functional groups. The inner circle shows the structure of the chloroplast: The large single copy (LSC), small single copy (SSC) and inverted repeat (IR) regions. The gray ring marks the GC content with the inner circle marking a $50 \%$ threshold. Asterisks mark genes that have introns.

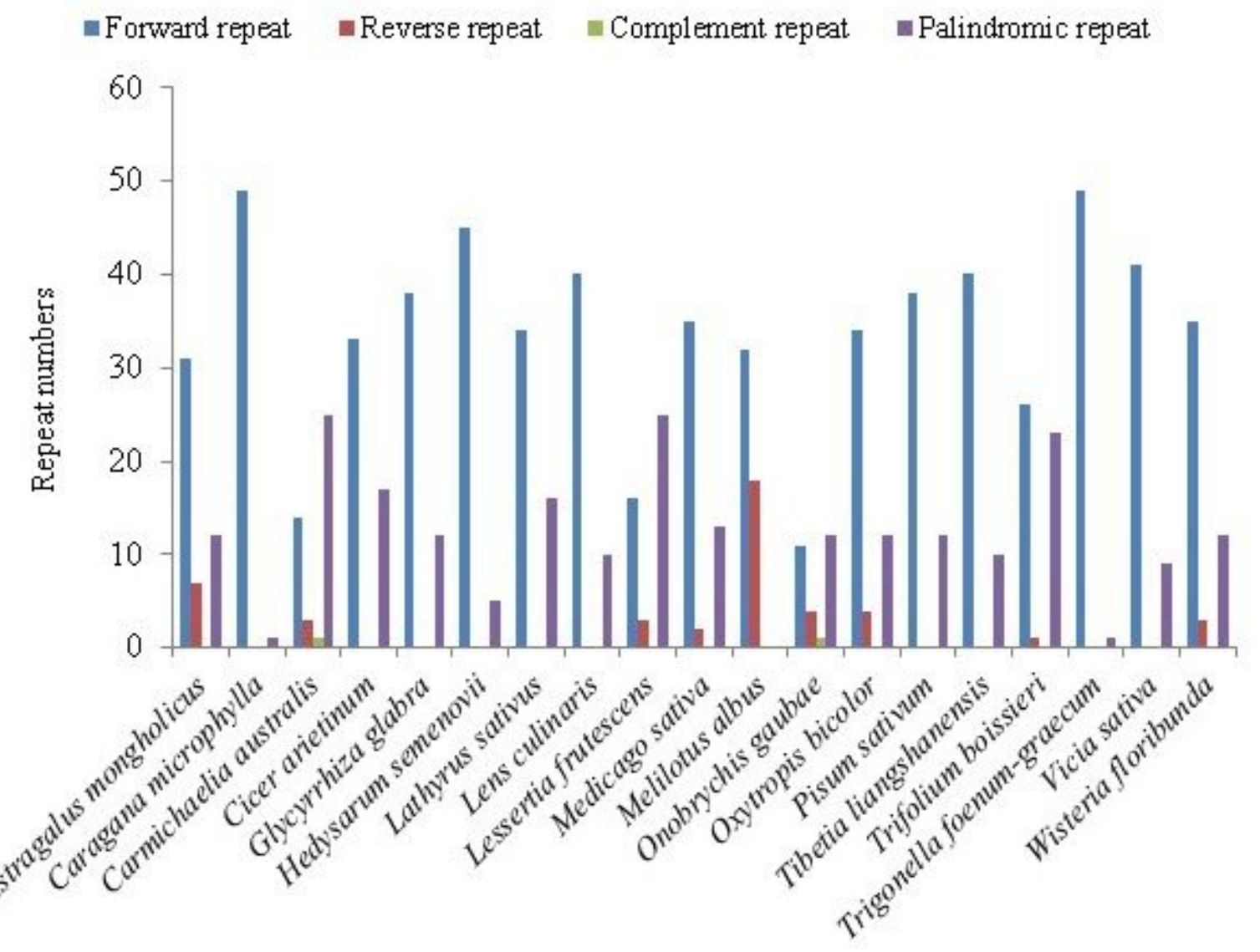

Figure 2

Analysis of repeated sequences in the IRLC species chloroplast genomes. 

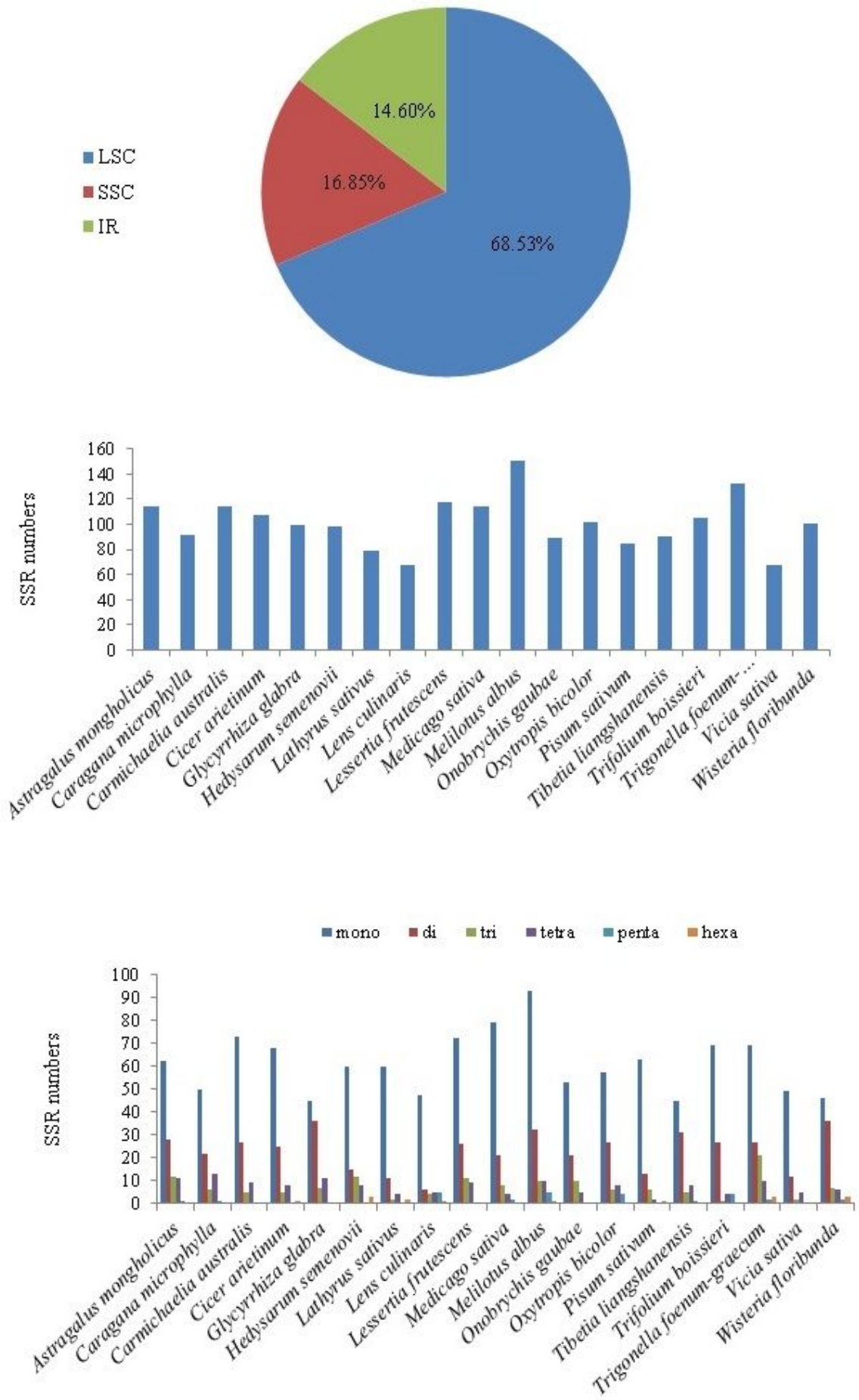

\section{Figure 3}

Analysis of perfect simple sequence repeats (SSRs) in the IRLC chloroplast genomes. (a) The frequency of identifed SSRs in large single-copy (LSC), inverted repeat (IR,) and small single-copy (SSC) regions; (b) The number of SSRs detected in the IRLC chloroplast genomes; (c) The number of SSR types detected in the IRLC chloroplast genomes. 


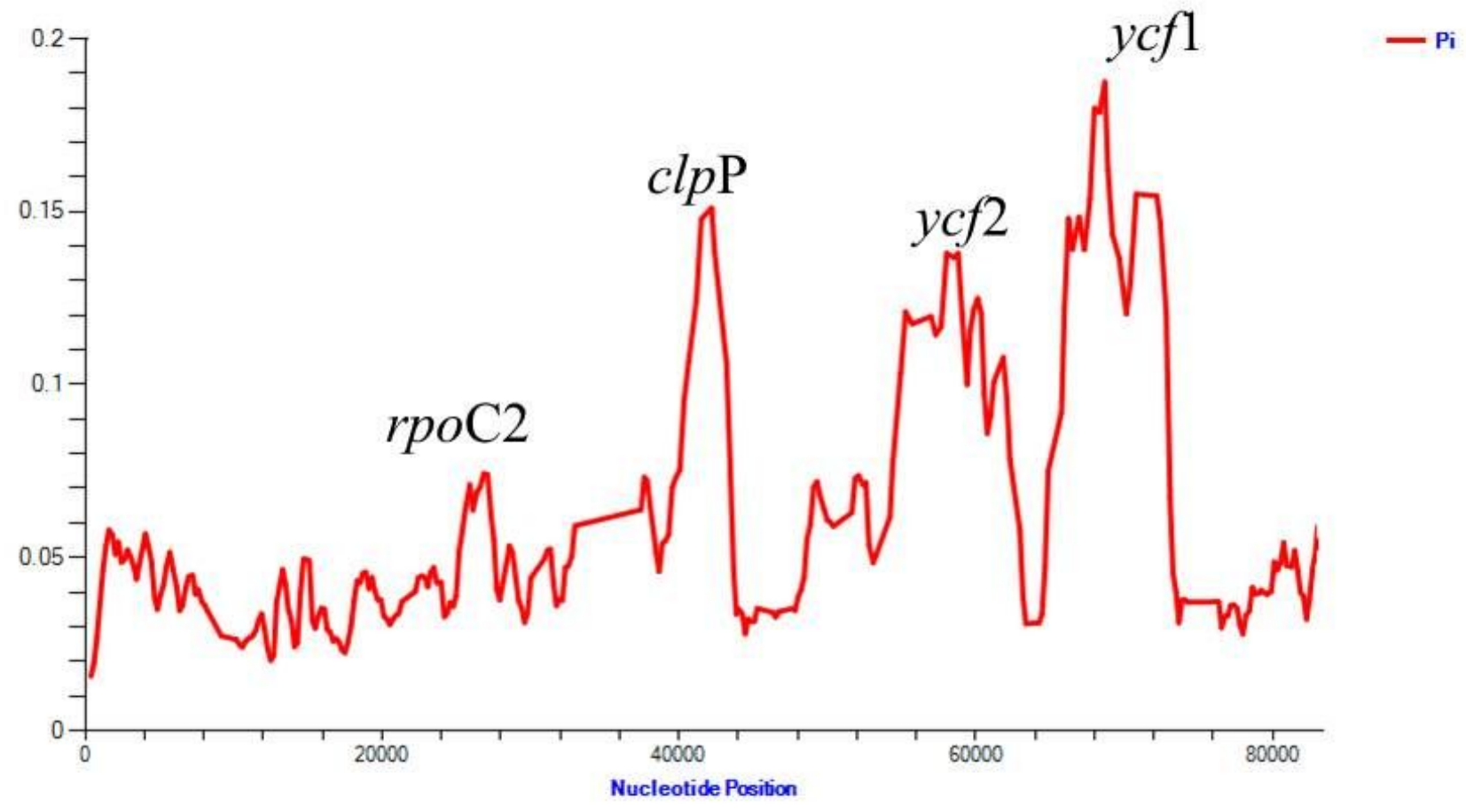

Figure 4

Nucleotide variability (\%) values among the IRLC species (using for coding regions). 

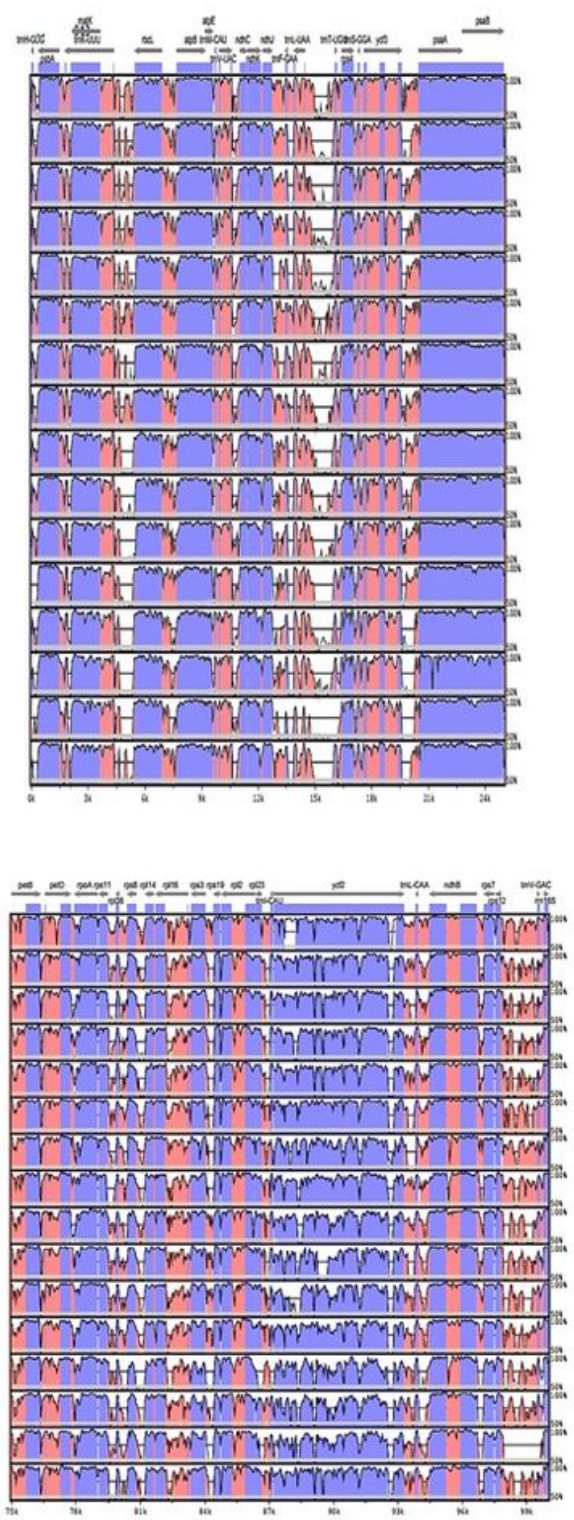
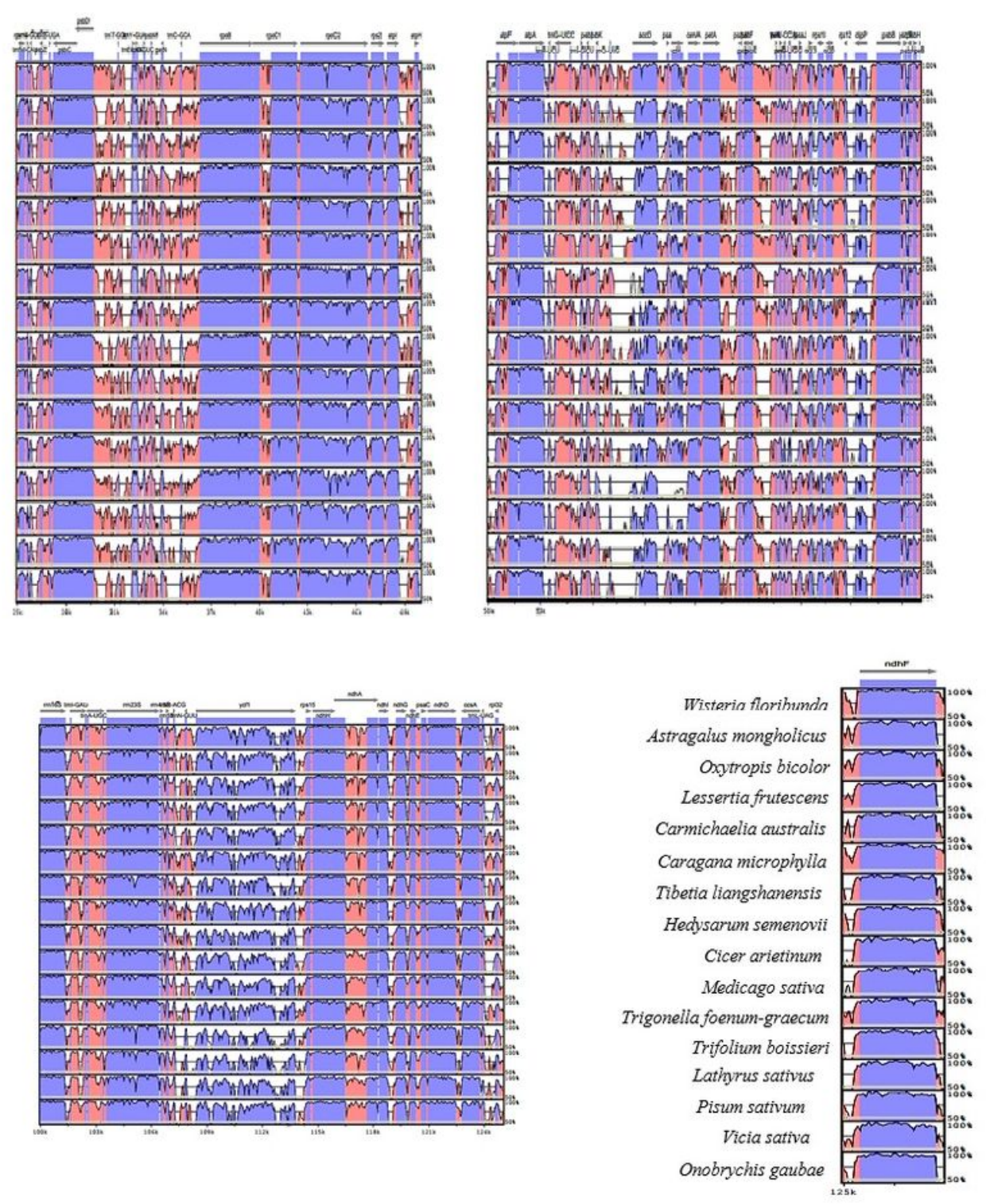

\section{Figure 5}

Sequence identity plot comparing the IRLC chloroplast genomes with Glycyrrhiza glabra as a reference by using mVista. Pink bars represent noncoding sequences (CNS), and white peaks represent genomic diferences. The y-axis represents the percentage identity (shown: 50-100\%).

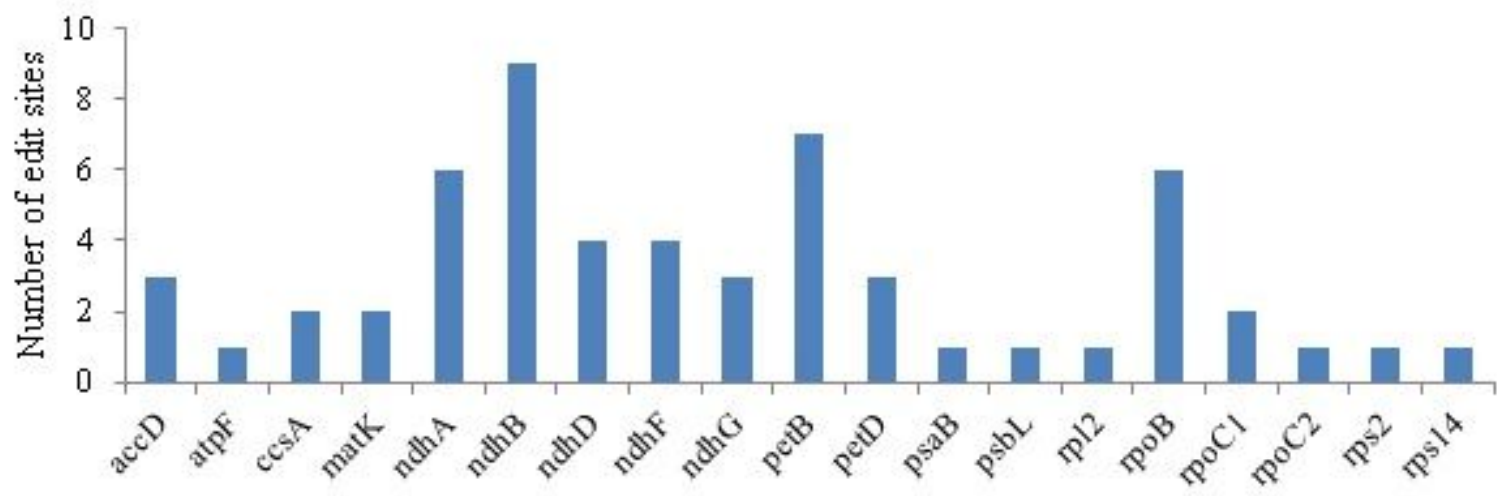


Figure 6

Distribution of number of RNA editing sites in the protein coding genes of the 0 . gaubae.

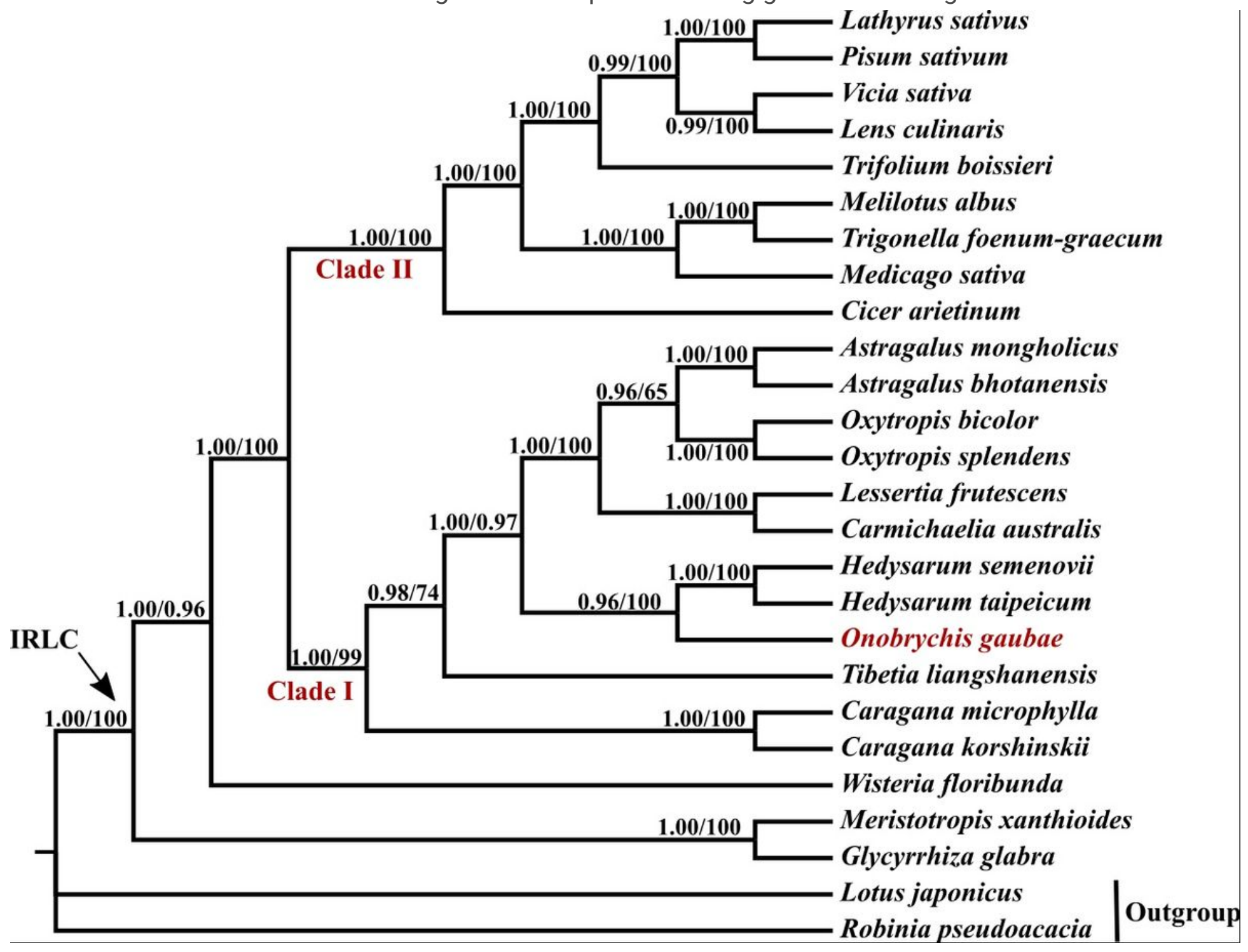

Figure 7

Fifty percent majority rule consensus tree resulting from Bayesian analysis of the 75 plastid genes of IRLC. The position of Onobrychis gaubae is shown in red. Numbers above branches are posterior probability and likelihood values, respectively.

\section{Supplementary Files}

This is a list of supplementary files associated with this preprint. Click to download.

- TableS4.xlsx

- Supplementaryinformation.pdf 